\section{Digital dentistry made easy}

Distributing products in over 120 countries, Planmeca's product range covers digital dental units, $2 \mathrm{D}$ and $3 \mathrm{D}$ imaging devices and comprehensive CAD/CAM and software solutions. Visit Planmeca at the British Dental Conference and Exhibition 2017 on stand B64 where they will have on show their latest CAD/CAM solution - PlanScan, for ultrafast intraoral scanning and the software suite that is the brains behind it all - Romexis.

Delegates will also have the opportunity to experience how the complete information on patient anatomy can be obtained in the minutest detail using the Planmeca ProMax 3D Classic unit. This intelligent and multipurpose X-ray unit provides digital panoramic, cephalometric and 3D imaging as well as 3D photos and 3D model scans. Planmeca look forward to introducing you to Planmeca's digital solutions and their benefits to you and your patient.

\section{Refreshed colours, same quality, same size}

TePe's interdental brushes have received an exciting colour refresh. The refreshed colours will remain consistent to their current sizing, and same high quality, but benefit from a modern new look.

Alongside the colour refresh, TePe's interdental brushes have also received two further updates. The patented soft neck, on the four smallest sizes, is now highlighted with a semi-transparent colour to further emphasise its highly flexible nature and a new look has been created for the cap, which functions as both a hygienic brush protector and smart handle extension.

TePe has been recommended by the dental profession in the UK for over 15 years. TePe offers a wide range of interdental brushes in nine different sizes to fit a variety of gaps, and are endorsed and recommended by $94 \%$ of dental hygienists in the UK. ${ }^{1}$

1. Eaton K A, Harris M, Ross M K, Arevalo C. A survey of dental hygienists in the United Kingdom in 2011. Part 1 - demographics and working patterns as dental hygienists. Br Dent J 2012; 213: E18.

\title{
Remineralisation now starts in the clinic
}

Until now there has been little the clinician can do to help patients reverse the early signs of erosive enamel wear. However, a new REGENERATE Professional Advanced Enamel Serum in-clinic remineralisation regime has now been launched by Unilever.

The process starts by making custom fit or stock dental trays for the upper and lower arches in the practice. These are used to apply the first of three applications of the REGENERATE Professional Advanced Enamel Serum - which takes only three minutes. The remaining two serum applications are completed at home by the patient over two subsequent days. Patients are advised to combine this with twice daily brushing with REGENERATE Enamel Science Advanced Toothpaste for best results. It is recommended that the Advanced Enamel Serum is used on a monthly basis.

Immediately after conducting a Basic Erosive Wear Examination (BEWE) to determine a patient's risk score for erosive wear, clinicians can integrate the serum as part of a preventive regime by applying the first application in-clinic and recommending the subsequent two applications at home.

Inspired by bone repair research, NR-5 technology contains two powerful ingredients - calcium silicate and sodium phosphate salts - which combine to form a fresh supply of enamel minerals, wrapping and integrating onto the teeth. ${ }^{1}$

Studies showed that the resulting products, REGENERATE Professional Advanced Enamel Serum combined with Advanced Toothpaste, can help reverse the early enamel erosion process. The REGENERATE Enamel Science system is clinically proven to provide superior re-hardening of acid-softened enamel in situ after three days compared with standard fluoride toothpaste.

Unilever has developed supporting practical materials for the dentist to diagnose enamel erosion including the BEWE Assist Guide along with patient educational materials. To purchase REGENERATE Professional Advanced Enamel Serum for the dental clinic contact CTS Dental Supplies by calling 01737 765400 or email: regenerate@cts-dental.com. www.regeneratenr5.co.uk/products/ advanced-toothpaste

1. Sun Y, Li X, Deng Y et al. Mode of action studies on the formation of enamel minerals from a novel toothpaste containing calcium silicate and sodium phosphate salts. J Dent 2014; 42 (Suppl 1): S30-38.

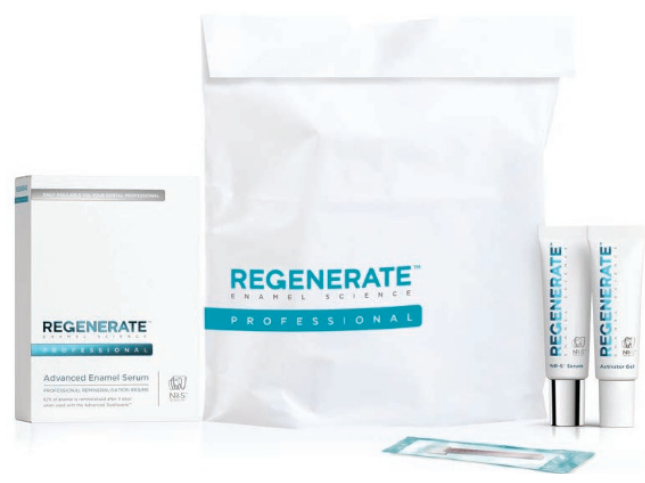

Digital, flexible and now, OPEN!

For over 30 years CEREC from Dentsply Sirona has provided a comprehensive and proven digital system for delivering restorations in a single visit. Now its applications are expanding and guided placement, restoration of implants and a variety of orthodontic solutions are also covered by this versatile system.

Offering clinicians even greater flexibility, the CEREC system has been opened up for the export of scan data in STL format. This creates new possibilities for clinicians by enabling data from CEREC digital impressions to be used in software products from other manufacturers, and allows them to send CEREC scans to a laboratory of their choice regardless of the software being used. Scan data can also be loaded into implant planning software from other manufacturers, providing clinicians with support in the preparation of implant procedures.

To find out more contact Dentsply Sirona on 08000723313 or visit www. dentsply.com/en-uk.

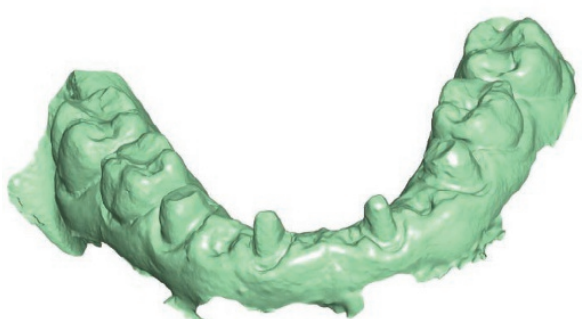

